\section{POSTER 525}

\section{The Medical Director Case Review: An Integral Part of an EMS Quality Improvement Program}

\section{Gratton MC}

Truman Medical Center

University of Missouri-Kansas City School of Medicine

Kansas City, Missouri, USA

Background: The Continuous Quality Improvement (CQI) process requires examining EMS performance to assess both individual and system strengths and weaknesses. Cases with potential or perceived problems are referred to the medical director for review.

Objective: To define prevalence and type of individual and system problems in a series of EMS medical director case reviews.

Design: Retrospective review of all cases referred for medical director review between 1 May 1991 and 30 April 1992. The review used implicit criteria. Patient outcome in each case was defined as either potentially positive (Pot Pos), neutral (Neu), or potentially negative (Pot Neg). Case review outcome (protocols followed, good judgment used, etc.) was defined as positive (Pos), neutral (Neu), or negative (Neg).

Setting: Public utility model, urban EMS system with approximately 55,000 calls and 40,000 transports per year.

Results: 202 cases were reviewed; 148 (73\%) involved only the ALS ambulance service. There were $9(4 \%)$ Pot Pos, 111 (55\%) Neu, 82 (41\%) Pot Neg patient outcomes. There were $10(7 \%)$ Pos, 62 (42\%) Neu, $76(51 \%)$ Neg review outcomes of cases involving just the advanced life support service. Patterns in problem areas were found including: $38(19 \%)$ documentation; $23(11 \%)$ destination; $20(10 \%)$ dispatch; $19(9 \%)$ radio medical control; $19(9 \%)$ lack of recognition of severity of illness; $17(8 \%)$ refusal of service; $15(7 \%)$ initial trauma care; 12 $(6 \%)$ respiratory distress. Patterns of provider problems were found. 63 paramedics were reviewed with 1 having $5 \mathrm{Neg}$ reviews, 2 having $4 \mathrm{Neg}$ reviews, 7 having $3 \mathrm{Neg}$ reviews.

Conclusion: Approximately $0.4 \%$ of calls were reviewed and $51 \%$ had a negative case review. Patterns of both system and provider problems were identified allowing for focused system improvement (i.e., revised protocols, education) and individual improvement (i.e., education, additional field training, officer assistance).

\section{POSTER 527}

\section{Badges, Blues and Body Armor: Have We Gone Too Far?}

\author{
Doran J, Lavery RF, Tortella BJ \\ The Division of Trauma and EMS Research \\ The New Jersey State Trauma Center, University Hospital \\ School of Medicine and Dentistry of New Jersey, \\ Newark, New Jersey, USA
}

Objective: To collect nationwide data on the relationship of EMS dress and provider-patient relationships, as it relates to similarities with local law enforcement agencies.

Design, Setting, and Participants: Single mail survey sent to training and operations officers of urban EMS systems in the 200 most populated United States cities.

Measurements and Main Results: There were 88 (45\%) surveys returned and evaluable. Seventeen $(19 \%)$ services reported their uniform mimicked local law enforcement and of these, $53 \%$ reported cases of assault and mistaken identity because of the similarity. Five $(7 \%)$ of the remaining services stated they purposely changed uniform style and color so not to look like law enforcement. Body armor was supplied by five $(6 \%)$ services and $13(15 \%)$ services reported that their personnel were compelled to wear body armor.

Conclusions: Presently, there is no national standard of dress for EMS providers and as far as we know, none at the state level. There is certain role confusion in regard to EMS appearance as services tend to mimic or even model law enforcement uniforms. It is difficult to establish patient-provider relationships when patients consider EMS providers to be police officers. An accurate history may be impossible to obtain, especially with regard to alcohol and drug usage and HIV status. It remains to be seen whether the present social climate in the United States has forced EMS providers to don body armor and color themselves more like law enforcement personnel and whether this impacts upon the provision of optimal patient care in the field. 